\title{
Simultaneous detection of salivary cortisol and cortisone using an automated high-throughput sample preparation method for LC-MS/MS
}

\author{
Ramisa Fariha ${ }^{\mathrm{a}}$, Mohannad Jabrah ${ }^{\mathrm{a}}$, Collin Hill ${ }^{\mathrm{b}}$, Adam Spooner ${ }^{\mathrm{a}}$, Prutha Deshpande ${ }^{\mathrm{a}}$, \\ Anubhav Tripathi ${ }^{\text {a,* }}$ \\ ${ }^{a}$ Center for Biomedical Engineering, School of Engineering, Brown University, 182 Hope Street, Providence, RI- 02912, USA \\ ${ }^{\mathrm{b}}$ PerkinElmer Inc., Waltham, MA, USA
}

\section{A B S T R A C T}

High-Performance Liquid Chromatography-Tandem Mass Spectrometry has emerged triumphant over the years as a reliable and high throughput clinical instrument to assess different metabolic irregularities. One of the latest applications of LC-MS/MS has been in the field of quantification of glucocorticoids, such as cortisol and cortisone from saliva, that can be an indicator of abnormalities such as Congenital Adrenal Hyperplasia (CAH), Cushing's Syndrome, and Addison's disease. We have developed and validated an LC-MS/MS-based assay for simultaneous detection of cortisol and cortisone in human saliva, which requires only $20 \mu \mathrm{L}$ of sample, to measure cortisol across a $0.5-70 \mathrm{ng} / \mathrm{mL}$ range, and cortisone across a $1.2-100 \mathrm{ng} / \mathrm{mL}$ range, respectively. The developed method exhibits linearity of $\mathrm{R}^{2}>0.99$, for both analytes, inclusive of both MRMs, and a percent coefficient of variation that is less than or equal to $20 \%$. Using dilute-and-shoot for sample preparation, we have exhibited sample accuracy of $100 \pm 20$ for the assay calibrators, and integrated Needle Wash Solvent Chemistry for minimal sample carryover, making it adaptable for crucial for potential diagnostic use. We have exhibited a method that is simplistic, specific, and highly automatable on liquid handling platforms, such as the JANUS ${ }^{\circledR}$ G3 Workstation. With our innovation, we have introduced a potential to test 81-unknown samples in singlicate within an on-deck plating time of fewer than four minutes. We performed additional verification studies, including an accelerated stability study and a freeze-thaw study showcasing the potential long-term usability of our proposed prototype kit. Overall, this work presents an optimized LC-MS/MS method with automated sample preparation that is ready to be utilized for cortisol-cortisone detection for clinical diagnostic contexts related to Cushing's Disease, among other adrenal and endocrine disorders.

\section{Introduction}

Glucocorticoids (GC), an important class of lipophilic, fat-soluble steroid hormones, regulate a wide variety of physiological functions. GCs modulate numerous processes including mood and cognitive function, growth, reproduction, and development, immune responses, as well as cardiovascular regulation [1-3]. As a result of this functional diversity, human GC nuclear receptors are expressed ubiquitously throughout the body and in all organs and tissues [4]. Two notable examples of endogenous glucocorticoids are cortisol $\left(\mathbf{C}_{\mathbf{F}}\right)$ and its inactive form, cortisone $\left(\mathrm{C}_{\mathrm{E}}\right)$.

Cortisol, widely known as the stress hormone, is synthesized in, and released from the adrenal cortex, resulting in elevated heart and respiratory rates. These are characteristic of the stress responses, which are triggered because of stressful stimuli that cause the hypothalamus activation of the hypothalamus-pituitary-adrenal (HPA) axis [5]. Elevated levels of cortisol trigger metabolic pathways in the liver, pancreas, and adipose tissue that are responsible for maintaining glucose and protein balance throughout the body [20]. High cortisol signals for increased gluconeogenesis and decreased glycogen synthesis and in the liver and for lipolysis in adipose tissue. Moreover, in the presence of high levels of cortisol, muscle cells decrease glucose uptake and consumption and shift towards increased protein degradation to provide the glucogenic amino acids necessary for gluconeogenesis [6,7].

Dysregulated levels of cortisol and cortisone may arise from abnormalities in glucocorticoid-activating enzymes such as $11 \beta$-hydroxylase and 21-hydroxylase deficiencies. The latter examples can take place in Congenital Adrenal Hyperplasia (CAH), as well as other inflammatory, psychiatric, and metabolic disorders $[8,9]$. Similarly, Cushing's disease and Addison's disease are both prominent adrenal disorders where clinical testing showcases clear signs of irregular levels of corticosteroids $[10,11]$. Circulating cortisol-cortisone can therefore function as biomarkers that allow for the detection and monitoring of chronic stresses, adrenal disorders, and more.

Herein, we have developed and validated a clinical method for metabolic irregularity assessment that utilizes a simple dilute-and-shoot protocol for the analysis of circulating cortisol-cortisone via Liquid Chromatography-Tandem Mass Spectrometry (LC-MS/MS) in saliva. LCMS/MS is a clinical analytical technique that has matured over the past decade, becoming the gold standard in many biochemical-diagnostics $[13,30]$. LC-MS/MS brings many advantages over other analyte detection alternatives, such as ELISA and immunostaining [27]. Among other advantages, LC-MS/MS provides the ability to accurately quantify analytes at low concentrations while maintaining high analyte specificity

\footnotetext{
* Corresponding author.

E-mail address: anubhav_tripathi@brown.edu (A. Tripathi).
} 
and exhibits superior sensitivity over other analytical techniques. This is critical in the context of cortisol-cortisone, where high cross-reactivity is frequently observed in immunoassays [28,29]. The proposed protocol was optimized to measure salivary analyte levels, as salivary cortisolcortisone levels are similarly indicative of free cortisol in plasma, serum, and urine [30]; however, saliva is easier to obtain and carries fewer disadvantages $[12,14,16]$. For example, attempts at quantifying freecortisol from urine samples encounter heightened matrix effects, due to high amounts of endogenous impurities, leading to ion suppression and loss of sensitivity [14,15]. Plasma samples require more complex sample preparation protocols, often necessitating a solid phase extraction step that reduces high-throughput capacity and increases processing costs per sample. Lastly, simultaneously measuring cortisol-cortisone from plasma may potentially yield misleading results [14], due to the $8: 1$ ratio of cortisol: cortisone in plasma, as opposed to the 1:2 ratio in saliva [20,21]. The $11 \beta$-hydroxysteroid dehydrogenase in the salivary glands aids the conversion of unbound cortisol to cortisone, leading to this vast difference in abundance. Therefore, saliva is highly indicated as a diagnostic matrix for rapid diagnosis of diseases affecting the adrenal glands via assessment of circulating glucocorticoids.

Plasma as a means for detecting free cortisol demands a sample preparation process of a higher degree of complexity due to an often-required solid phase extraction procedure that reduces the highthroughput capacity of the proposed method, as well as increases the cost of processing per sample. Lastly, measuring cortisol from plasma may potentially yield misleading results due to its measurement of the total amount of cortisol, including bound cortisol [14]. Ultimately, this encourages the utilization of saliva as a means for rapid recognition, diagnosis, and treatment of diseases affecting the adrenal glands by accurately evaluating circulating glucocorticoid levels.

The present work focuses on optimizing streamlined sample preparation and LC-MS/MS analysis method that avoids the need for errorbound extraction steps (liquid-liquid extraction, solid-phase extraction, etc.) often found in other protocols. This reduces the complexity of the sample preparation process, allowing for a more streamlined and highthroughput automation using conventional robotic liquid handlers, with minimal needed human interaction. Overall, as shown in Table 1, the methodology of this work focuses on a rationalized and simplified approach, while maintaining the linearity of analyte detection with relevant clinical ranges of disease. The proposed method exhibits an excellent range of linearity for established salivary cortisol for $\mathrm{CAH}$, dexamethasone suppression, ACTH stimulation, Syndrome of Apparent Mineralocorticoid Excess (SAME), and other metabolic disorders [25,26]. The proposed protocol has the potential to be utilized as a high-throughput research-only kit, that can measure the free cortisol-cortisone in human saliva with great precision and accuracy using only a $20 \mu \mathrm{L}$ patient sample volume, across the range of 0.5 to $70 \mathrm{ng} / \mathrm{mL}\left(\mathrm{C}_{\mathrm{F}}\right)$ and 1.2 to 100 $\mathrm{ng} / \mathrm{mL}\left(\mathrm{C}_{\mathrm{E}}\right)$ distributed across six calibrators and low and high-Quality Controls (QCs).

\section{Experimental}

\subsection{Chemicals and reagents}

Baker Analyzed HPLC grade water, acetonitrile, and methanol ( $>99.9 \%$ purity) were obtained from VWR for the preparation of calibrators, test samples, and the equilibration of the chromatographic column to avoid particulate matter that might potentially affect LC-MS/MS performance. Ampules of primary analytes of study - Cortisol $\left(\mathrm{C}_{21} \mathrm{H}_{30} \mathrm{O}_{5}\right.$, $\mathrm{MW}=362.46 \mathrm{amu})$, Cortisone $\left(\mathrm{C}_{21} \mathrm{H}_{28} \mathrm{O}_{5}\right.$, MW= $\left.360.44 \mathrm{amu}\right)$, Cortisol$\mathrm{D}_{4}$ (internal standard) $\left(\mathrm{C}_{21} \mathrm{H}_{26} \mathrm{D}_{4} \mathrm{O}_{5}, \mathrm{MW}=366.48 \mathrm{amu}\right)$ and Cortisone${ }^{13} \mathrm{C}_{3}$ (internal standard) $\left(\mathrm{C}_{18}{ }^{13} \mathrm{C}_{3} \mathrm{H}_{28} \mathrm{O}_{5}\right.$, MW= $363.42 \mathrm{amu}$ ) were purchased from Cerilliant Corporation (Round Rock, TX, USA). Reagent grade formic acid was obtained from Sigma-Aldrich. Utak SMX Oral Fluid (Valencia, CA) was used as the base matrix to create the calibrators and QCs.

\subsection{Preparation of calibrators, quality controls, and stock solutions}

The cortisol and cortisone stock solutions were initially used to prepare spike solutions, using a two-step dilution method. This was done to ensure a $2.5 \%$ or less volume percentage of methanol across all calibrators and QCs, yielding five spike solutions for cortisol and six for cortisone, respectively. The Utak SMX Oral Fluid was then spiked using these spike solutions to create the calibrators and QCs, covering a concentration range of $0.5-70.0 \mathrm{ng} / \mathrm{mL}\left(\mathrm{C}_{\mathrm{F}}\right)$ and $1.2-100.0 \mathrm{ng} / \mathrm{mL}\left(\mathrm{C}_{\mathrm{E}}\right)$. Calibrators, as well as QCs, were aliquoted and stored at $-80^{\circ} \mathrm{C}$ before starting any system suitability study. Additionally, a temperature-controlled centrifuge step $\left(4^{\circ} \mathrm{C}\right.$, at $\left.4600 \mathrm{RPM}\right)$ was added to the protocol and investigated as a pre-treatment condition to emphasize solid particulate pellet formation, to prevent system clogging after injection of calibrators and QCs in LC-MS/MS.

Daily Working Solution (DWS) was prepared in a 50:50 (v/v) solution of HPLC water to HPLC methanol, with the final concentration at $1.7 \mathrm{ng} / \mathrm{mL}$ and $1.3 \mathrm{ng} / \mathrm{mL}$ for cortisol and cortisone, respectively. The DWS was stored at $-20^{\circ} \mathrm{C}$ and was warmed to room temperature prior to each assay.

\subsection{LC-MS/MS conditions and optimized parameters}

The cortisol and cortisone analytes were diluted 10,000- and 1000fold respectively, in HPLC-grade methanol before being directly infused into the MS to quantify the parent mass, quantifier fragment, and qualifier fragment masses. This was also repeated with the corresponding analyte internal standards, diluted 100 folds in HPLC-grade methanol. The compound transition data, together with the Entrance Voltage (EV), Collision Cell Energy (CC), and Collision Cell Lens 2 (CCL2) values obtained from Multiple Reaction Monitoring (MRM) optimization, are available in Supplemental Table S1.

After MRM optimization, the nebulizer gas pressure, source temperature, HSID (hot surface-induced desolvation) temperature, and drying gas pressure were also optimized to obtain the best relative intensity for both Q3 masses of the analytes and their internal standards. After the Q1 and Q3 masses were detected, the EV, CC, and CCL2 values were re-optimized for the best signal of the analyte quantifier, qualifier, and internal standards. The LC-MS/MS system used was a PerkinElmer QSight ${ }^{\circledR}$ 200-series Triple Quadrupole Mass Spectrometer, equipped with an Electron Spray Injection (ESI) source in using positive ion mode. Integrated to the MS was a QSight LX50 Solvent Delivery Module, QSight LX50 Precision Sampling Module, and QSight ${ }^{\circledR}$ LX-50 Column Stability Module for the column temperature control. The column used was a PerkinElmer Brownlee ${ }^{\circledR}$ SPP C-18 column $(2.7 \mu \mathrm{m}$, C18, $90 \AA, 4.6 \times 75 \mathrm{~mm}$ ), set to be $40^{\circ} \mathrm{C}$ for the entire assay run, with mobile phase A (inorganic) and mobile phase B (organic) according to an optimized flow profile to the flow on table 4 . The phase was designed with a linear hold, ranging from $100 \%$ A to $95 \%$ B, and back to $100 \%$ A (phase-flow design is available in Supplemental table S2). For improved carryover mitigation, the Precision Sampling Module needle was put through weak-strong-weak solvent wash cycles, where the weak solvent was 70:30= water: acetonitrile solution and the strong solvent was 100\% HPLC acetonitrile. The volume was kept at $250 \mu \mathrm{L}$ for both wash solvents during the wash cycles. Sample delivery was carried out through a $250 \mu \mathrm{L}$ syringe, connected to a $10 \mu \mathrm{L}$ needle and a $20 \mu \mathrm{L}$ loop, with triple quadruple MS operation at a nebulizer pressure of 200 psi, electrospray voltage of 5850 volts, and a source temperature of $425^{\circ} \mathrm{C}$. This produced runs of a total of 2.5 minutes for each sample for simultaneous analyte detection. Afterward, all subsequent chromatographic analyses were performed on the Simplicity ${ }^{\mathrm{TM}}$ $3 Q$ (version 1.9) software designed specifically for the QSight instrument.

We performed a phase test with $100 \mathrm{ng} / \mathrm{mL}$ concentration of cortisol and cortisone, dissolved in ratios of organic to inorganic solvents ranging from organic $:$ inorganic $=95: 5$ to $5: 95$. In addition, 
Table 1

a) A comparative study of our method against existing literature. It is important to note that our proposed method targets a more clinically relevant range, unlike the other studies.

b) Reference range for targeted metabolic disorders for $\mathrm{C}_{\mathrm{F}}$ and $\mathrm{C}_{\mathrm{E}}$, pertaining to genders assigned at birth and infants.

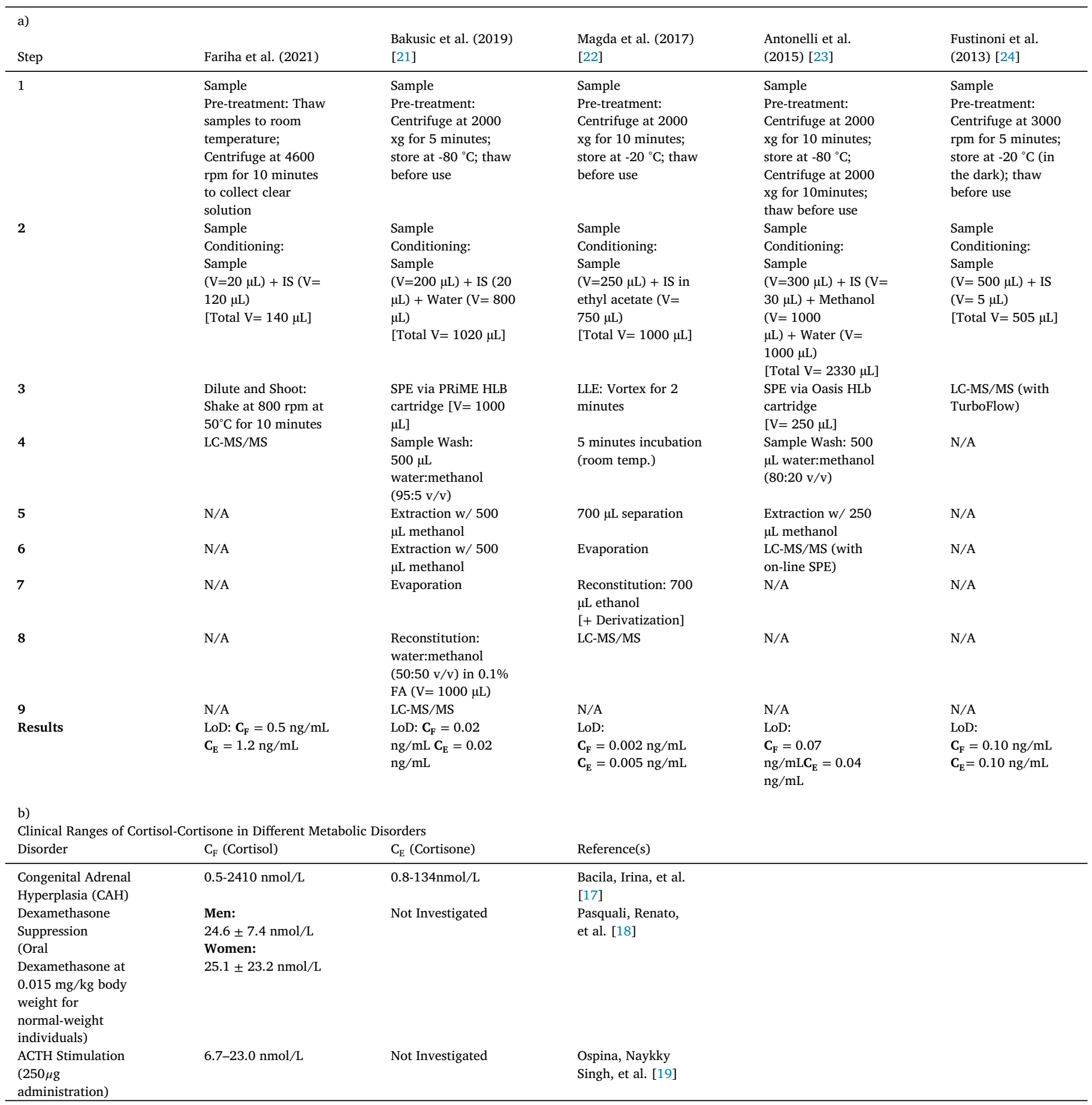

we performed a 4-parameter test (data not reported) to test the solubility of the analytes in organic solvents (acetonitrile and methanol) and their absolute intensities with respect to two different organic solvents used for mobile phase B. Acetonitrile as mobile phase B yielded the strongest relative signal for each calibrator level and methanol was determined to be a better solvent for dissolving the analytes. The formic acid level was determined to be $0.1 \%$ for both mobile phases and the flow rate was held steady at $0.6 \mathrm{~mL} / \mathrm{min}$ throughout the experiment.

\subsection{Sample preparation}

Calibrators, QCs, and samples were thawed at room temperature, followed by centrifuging at 4600 RPM for 10 minutes at $4^{\circ} \mathrm{C}$. Avoiding the particulate pellet at the bottom, $20 \mu \mathrm{L}$ of the clear solution from the top was added to each well on a large volume 96-well plate, followed by the addition of $120 \mu \mathrm{L}$ of the DWS to each well. HPLC water was used as blanks. The plate was properly sealed and shaken using the TriNEST ${ }^{\mathrm{TM}}$ microplate shaker at 800 RPM for ten minutes at a controlled temperature of $50^{\circ} \mathrm{C}$, before inserting the 96 -well plate into 
Table 2

(Top: Cortisol- Quantifier; Bottom: Cortisone- Quantifier) \% CV was used as a measure for precision, determined using 3 replicates per level each day, over a 6-day period ( $n=18$ per level). Samples were found to be within $12 \%$ or less for intraday and interday measurements, inclusive of both MRMs for both analytes. The average $\%$ accuracy for all calibrators was found to be $100 \pm 20$, inclusive of both MRMs for both analytes. These values fall well within our $\leq 20 \%$ cut-off value.

\begin{tabular}{cccc}
\hline Calibrator Level & $\boldsymbol{\mu}_{\overline{\boldsymbol{x}}} \pm \boldsymbol{\sigma}(\mathrm{in} \mathrm{ng} / \mathrm{mL})$ & $\% \mathrm{CV}$ & \% Accuracy \\
\hline L1 & $0.55 \pm 0.05$ & 8.56 & 110 \\
\hline L2 & $2.58 \pm 0.14$ & 5.61 & 86 \\
\hline L3 & $13.44 \pm 0.55$ & 4.10 & 103 \\
\hline L4 & $21.12 \pm 0.88$ & 4.16 & 101 \\
\hline L5 & $30.19 \pm 0.90$ & 2.99 & 101 \\
\hline L6 & $69.61 \pm 2.00$ & 2.87 & 99 \\
\hline
\end{tabular}

\begin{tabular}{cccc}
\hline Calibrator Level & $\boldsymbol{\mu}_{\overline{\boldsymbol{x}}} \pm \boldsymbol{\sigma}$ (in $\left.\mathbf{n g} / \mathrm{mL}\right)$ & $\%$ CV (Inter-Assay) & \% Accuracy \\
\hline L1 & $1.13 \pm 0.13$ & 11.30 & 94 \\
L2 & $5.72 \pm 0.39$ & 6.76 & 95 \\
\hline L3 & $15.93 \pm 0.55$ & 3.44 & 106 \\
\hline L4 & $43.83 \pm 1.75$ & 3.98 & 104 \\
\hline L5 & $52.07 \pm 0.95$ & 1.82 & 104 \\
\hline L6 & $95.51 \pm 2.88$ & 3.02 & 96 \\
\hline
\end{tabular}

the LC-MS/MS autosampler with a Rapid EPS plate seal (BioChromato, Japan) for analysis.

\subsection{Accuracy, precision, and linearity}

In order to assess the validity of the proposed LC-MS/MS method, testing of several parameters indicating the assay sensitivity was performed. This was done in a 6-day study, where each calibrator level and QCs were analyzed in triplicates. To confirm method linearity over the specified target range, calibration curves were evaluated from the analysis of the six different calibrator levels on Simplicity 3Q (Version 1.9) software. A standard curve was generated using linear regression with $\frac{1}{x}$ weighting, where the analyte-to-IS ratios were compared against known calibrator concentrations. The series inter-assay linearity was evaluated from this linear regression, with the reported R-Square value as the primary assessment of assay linearity.

Similarly, assay precision was measured as the percent coefficient of variation $(\% \mathrm{CV})$, which was obtained from the aforementioned calibration curves for each level within run, between run, and total. Sample accuracy was obtained from the automatically reported analysis from Simplicity 3Q software and averaged for all levels across all runs. For each plate, quality control samples (low $=3 \mathrm{ng} / \mathrm{mL}$ for cortisol, $6 \mathrm{ng} / \mathrm{mL}$ for cortisone; high $=30 \mathrm{ng} / \mathrm{mL}$ for cortisol, $50 \mathrm{ng} / \mathrm{mL}$ for cortisone) were run together with the calibrators for further validation of the inter-assay precision.

\subsection{Freeze-thaw}

Information on the developed cortisol-cortisone quantification kit's stability was obtained by performing freeze-thaw testing over multiple cycles. Freeze-thaw stability testing was performed on a full prototype kit, comprising the six cortisol-cortisone calibrators, the two quality controls, as well as the internal standard. Cortisol-cortisone kits were produced and subjected to six freeze-thaw cycles via transfer from one of two cold reservoirs $\left(-20^{\circ} \mathrm{C}\right.$ and $\left.-80^{\circ} \mathrm{C}\right)$ to room temperature and measuring concentration changes of cortisol-cortisone over the six freeze-thaw cycles by analyzing each calibrator level in triplicate.

\subsection{Assessing sample carry-over}

To confirm a minimal sample carryover for the proposed sample preparation and LC-MS/MS methodology, the highest calibrator level was injected into the system, followed by triplicate injections of the lowest calibrator level. Any deviation in the measurement of the cortisolcortisone quantities in the lowest level was observed and recorded, with sample carryover accounting for any significant changes in the analyte concentrations due to the first injection of the highest calibrator concentration.

\subsection{Evaluating stability of prepared standards}

To obtain stability information on the proposed cortisol-cortisone diagnostic kit, we performed an accelerated 14-day accelerated stability 


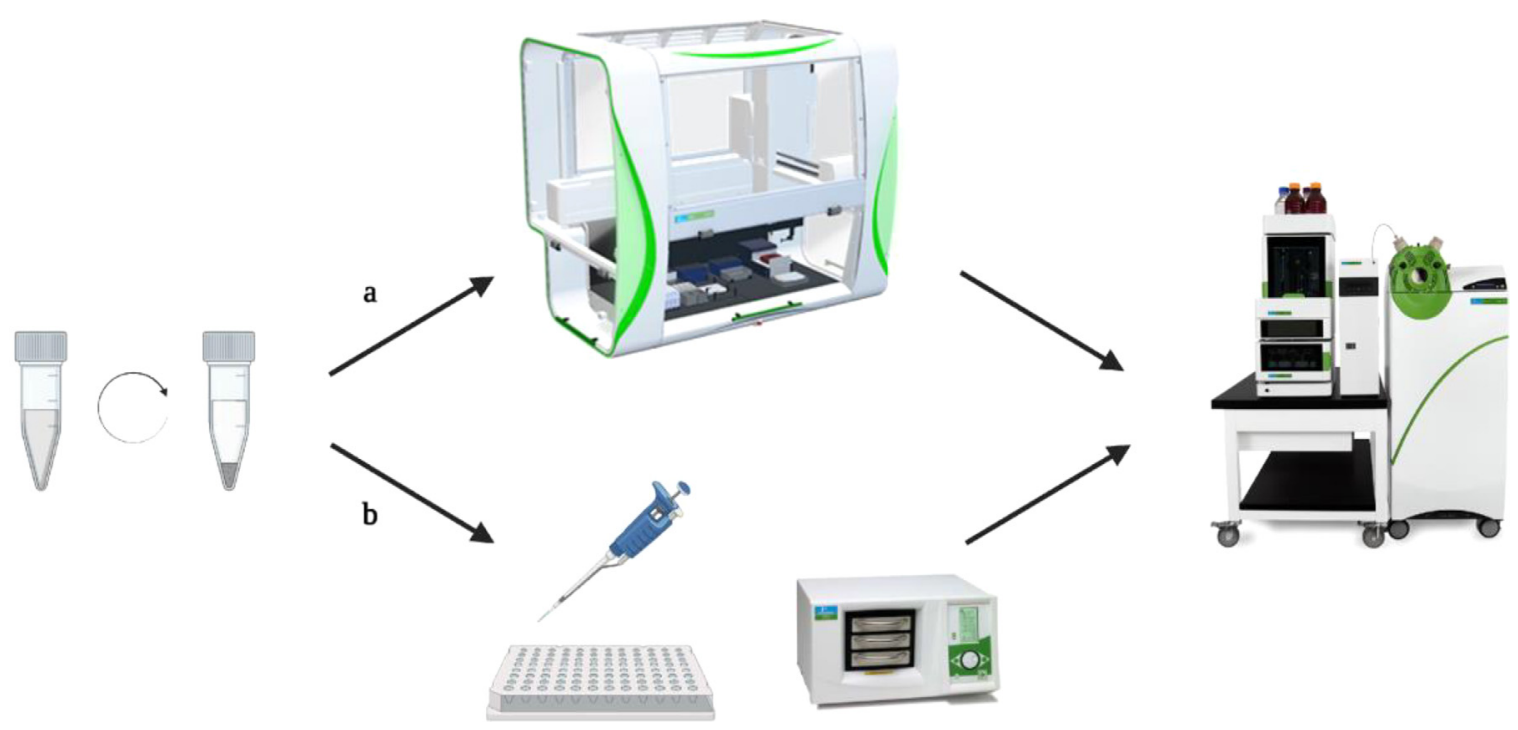

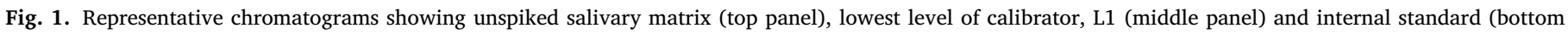

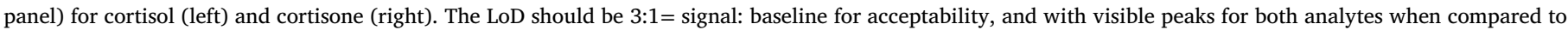
blank, our LoD meets this criterion. The result yielded by the blank matrix was almost identical to HPLC water.

study in compliance with the Clinical and Laboratory Standards Institute (CLSI) guidelines for stability testing of in vitro diagnostic reagents. Kit calibrators were subjected to extreme temperature changes by being moved across different storage temperatures. Two sets of calibrators were tested for stability, with one being transferred from $-80^{\circ} \mathrm{C}$ to $-20^{\circ} \mathrm{C}$, and another from $-20^{\circ} \mathrm{C}$ to $+30^{\circ} \mathrm{C}$; calibrators of the same level were transferred in duplicates. Transfers were staggered and done every two days, for a total of seven transfers over the total period of 14 days.

After the last transfer on the 14th day, samples were harvested and tested against fresh samples to confirm the threshold of stability. Following this accelerated stability study and the confirmation of analyte retention after sample stressing, the following equation (derived from Arrhenius' rate equation) was utilized to obtain an estimate for calibrator stability without any degradation. This derivation utilizes the tendegree rule, where the rate of reaction is said to double for every ten degrees Celsius change in temperature.

Stable Days $=($ Days in high temperature $) \times\left(2^{\frac{\Delta \text { Temperature }}{10}}\right)$

Equation 1. Modified Arrhenius' rate equation for measurement of sample stability.

\subsection{Automation of sample preparation protocol}

To further adapt the cortisol-cortisone quantification kit to clinical applications, the developed sample preparation method was automated and tested for improved high-throughput capacity using the JANUS@ G3 Workstation (PerkinElmer Corp., MA, U.S.). The sample preparation process previously described was adapted onto the JANUS ${ }^{\circledR}$ workstation using JANUS ${ }^{\circledR}$ Application Assistant and WinPREP ${ }^{\circledR}$ for JANUS ${ }^{\circledR}$, via the deck arrangement illustrated in Supplemental Fig. 1. To determine the efficacy of this automated sample preparation method, manually prepared samples were compared against automated ones with regards to precision and accuracy.

The JANUS ${ }^{\circledR}$ G3 Workstation is used to automate the sample preparation. The viscosity of the samples was found comparable to water for all standard levels of cortisol-cortisone in oral fluid. Using this information, performance files for liquids with viscosity near or at that of water were used; the details of the performance files for the Blowout Mode, and the Waste Mode can be found in Supplemental Table S3(a) and S3(b), respectively 7 ). These performance files ensure that the aspirations and dispenses are accurate and precise each time. Depending on our application and plate layouts, we utilized both Blowout and Waste
Mode performance files. For a singlicate dispense, such as the calibrators, we used the Blowout Mode, whereas, for dispenses per aspirate $\geq$ 2 , such as an unknown sample to be tested, we used the Waste Mode. The design of this automation protocol was to maximize the number of unknown samples that could be quantified accurately per assay.

The DWS containing methanol required the addition of a pipette-tip prewetting step and the introduction of transport air gaps into the tip to combat volatile liquid loss. By aspirating and dispensing volume into and out of the pipette tip, the tip becomes 'prewet'; this action saturates the interior of the pipetting tip with vapors from the solution. When the final aspiration is made, the interaction between the tip and the volatile liquid will no longer create vapor build-up, which is a significant cause of dripping. In addition, air gaps assisted in minimizing the leakage. Aspirating $10 \mu \mathrm{l}$ of air after initial aspiration and each subsequent dispense was beneficial in combating the dripping of volatile DWS from the pipetting tips.

\section{Results and discussion}

\subsection{Blank matrix study}

For every assay run, L0 (unspiked oral fluid) was injected in triplicate into LC-MS/MS system with the goal of ensuring minimal signal noise. The calculated concentration for both cortisol and cortisone were $<0 \pm 0.008 \mathrm{ng} / \mathrm{mL}$, or no peak was observed, demonstrating the absence of either analyte in the blank matrix. The criteria of acceptance for LoD is a signal-to-baseline ratio of 3-to-1, which our LoD met for both cortisolcortisone (Fig. 2).

\subsection{Phase test and 4-parameter test}

The main purpose of performing the phase test was to obtain the lowest percentage of organic solvent at the end of the final step of the sample preparation, to yield a symmetrical chromatogram with no peak distortion and good signal intensity. An ideal peak should yield a symmetrical Gaussian profile (Equation 2), with minimal peak broadening, exhibiting the highest column efficiency.

$y=\frac{1}{\sigma \sqrt{2 \pi}} \exp \left[-\frac{1}{2}\left(\frac{x-\bar{x}}{\sigma}\right)^{2}\right] ;$

where $\sigma=$ standard deviation, $\bar{x}=$ mean and $x=$

$\bar{x}$ at the axis of symmetry 


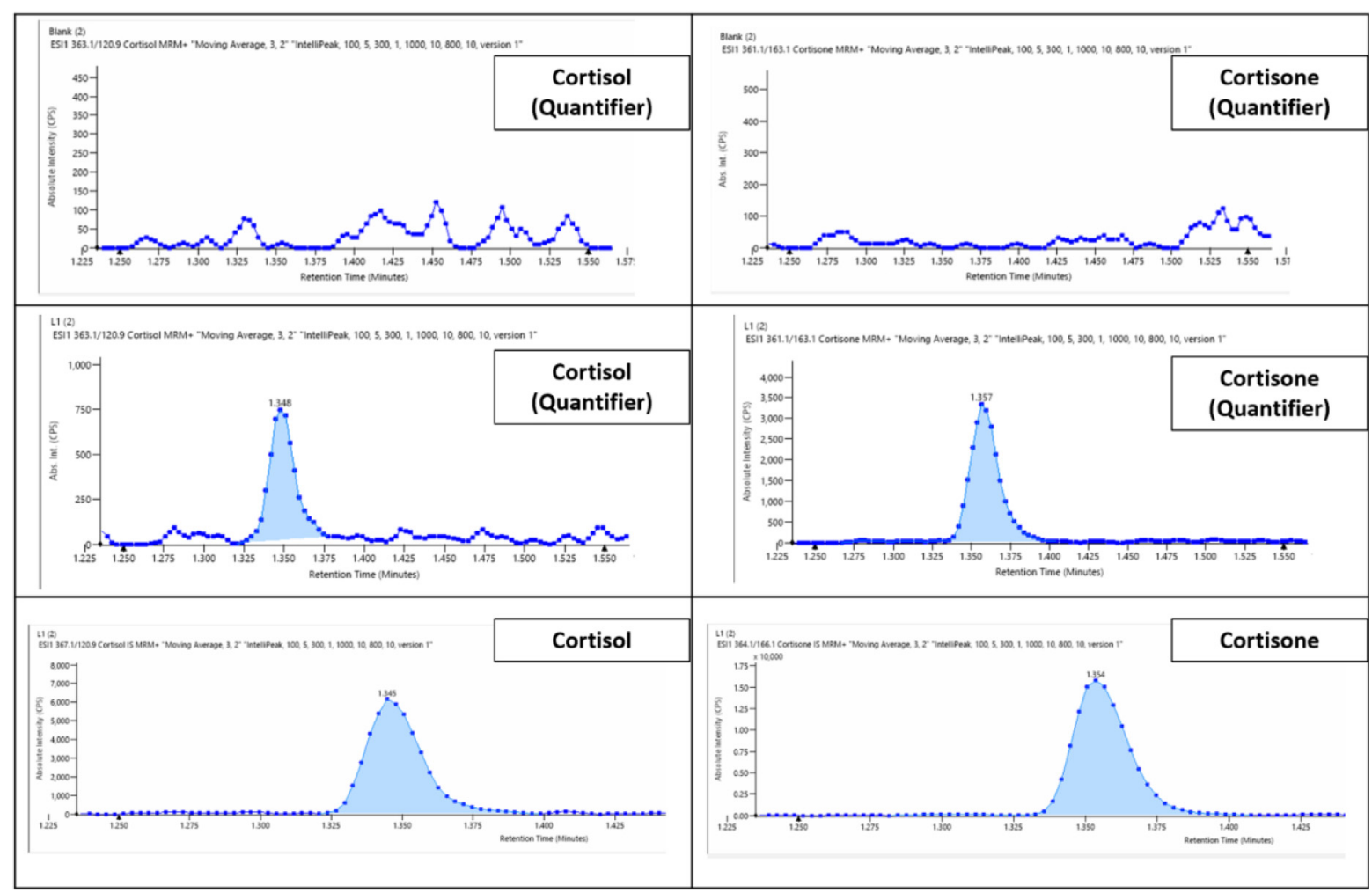

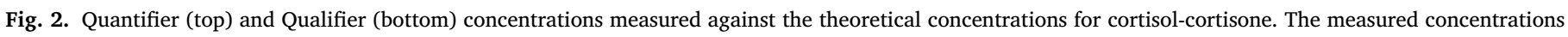
were used in the generation of the standard curves for all further tests.

Equation 2. Gaussian distribution for chromatogram with no peak distortion.

This meant that any percentage of organic and inorganic that produced chromatograms with peak fronting and peak tailing were not considered. Even though the water : acetonitrile $=55: 45$ ratio gave the best peak shape, upon inspecting the individual MRM intensities, we observed that water : acetonitrile $=35: 65$ yielded the highest relative intensity for both the analytes . That said, assessing the signal-to-noise ratio for each analyte was also crucial to the method development process. Both analytes (for quantifier and qualifier) for each inorganic : organic ratio were tested in triplicates and we averaged the MRM intensity and signal-to-noise ratio for each solvent ratio to conclude that water : acetonitrile $=60: 40$ resulted in a Gaussian peak with the best MRM intensity, along with a high signal, and low noise (selected simplified chromatograms are available in Supplemental Fig. S2).

\subsection{True concentration test (QC Study)}

Because the calibrators were prepared using commercially available cortisol-cortisone in methanol, there was a possibility of the concentration of the actual stock solution not matching the stated theoretical value. Since cortisol and cortisone do not have NIST (National Institute of Standards and Technology) certified material, we had to establish a true concentration of calibrators and quality controls for each manufacturing batch, assuming that the stock solution concentrations measured exactly at $1 \mathrm{mg} / \mathrm{mL}$. As each set of calibrators and quality controls were prepared, each level was analyzed in sextuplets and averaged to obtain the 'true concentration', to avoid error propagation in precision measurements for the following experiments. Fig. 6 shows both quantifier and qualifier mass data for both analytes and the 'true concentrations' against the theoretical concentrations.

\subsection{Linearity, precision, and accuracy}

Cortisol and cortisone calibrators both reported intra and inter-assay precision $(\% \mathrm{CV})$ measured at $\leq 20 \%$. Moreover, for further validation of the results, inter-assay \% $\mathrm{CV}$ of the QCs of both cortisol-cortisone both reported measurements of $\leq 10 \%$. Generally, each lab sets the precision threshold for their LC-MS/MS studies, with $\pm 15-20 \%$ being standard. Given the absence of NIST-certified materials for cortisol and cortisone, it is challenging to standardize any clinical assay. Hence, we utilized our True Concentration Test to establish the QCs and calibrators such that they can be used to establish the baseline and quantify any QCs for future calibrator manufacturing. The accuracy for the calibrators was within the $100 \pm 20 \%$ range for both analytes (Table 2 ). This establishes our method, together with the use of the UTak salivary matrix, to be implemented in clinical laboratory settings for the generation of standard curves for the said glucocorticoid measurements in patient samples. The $\mathrm{R}$-square values were taken as a measure of the linearity, generated in Simplicity $3 \mathrm{Q}$ software using linear regression. Linearity was calculated by plotting the mean measured analyte concentrations, as a function of the corresponding known enrichment levels and performing linear regression analysis. For both cortisol and cortisone, the R-square value was greater than 0.99 for both MRMs across all 6 days (Supplemental Table S4). In addition, the precision, linearity, and accuracy studies were performed for cortisol-cortisone quantification kits in two storage temperatures: $-80^{\circ} \mathrm{C}$ (reported) and $-20^{\circ} \mathrm{C}$ (not reported). The results obtained for both temperatures were very similar, with the $\% \mathrm{CV} \leq 20 \%$ for both cortisol and cortisone (inclusive of both MRMs) at both storage temperatures, with $\mathrm{n}=18$ per level across the study.

\subsection{Freeze-thaw stability}

The concentration of each calibrator and QCs (low and high) for both sets of freeze-thaw cycles (from $-80^{\circ} \mathrm{C}$ to room temperature and $-20^{\circ} \mathrm{C}$ to 


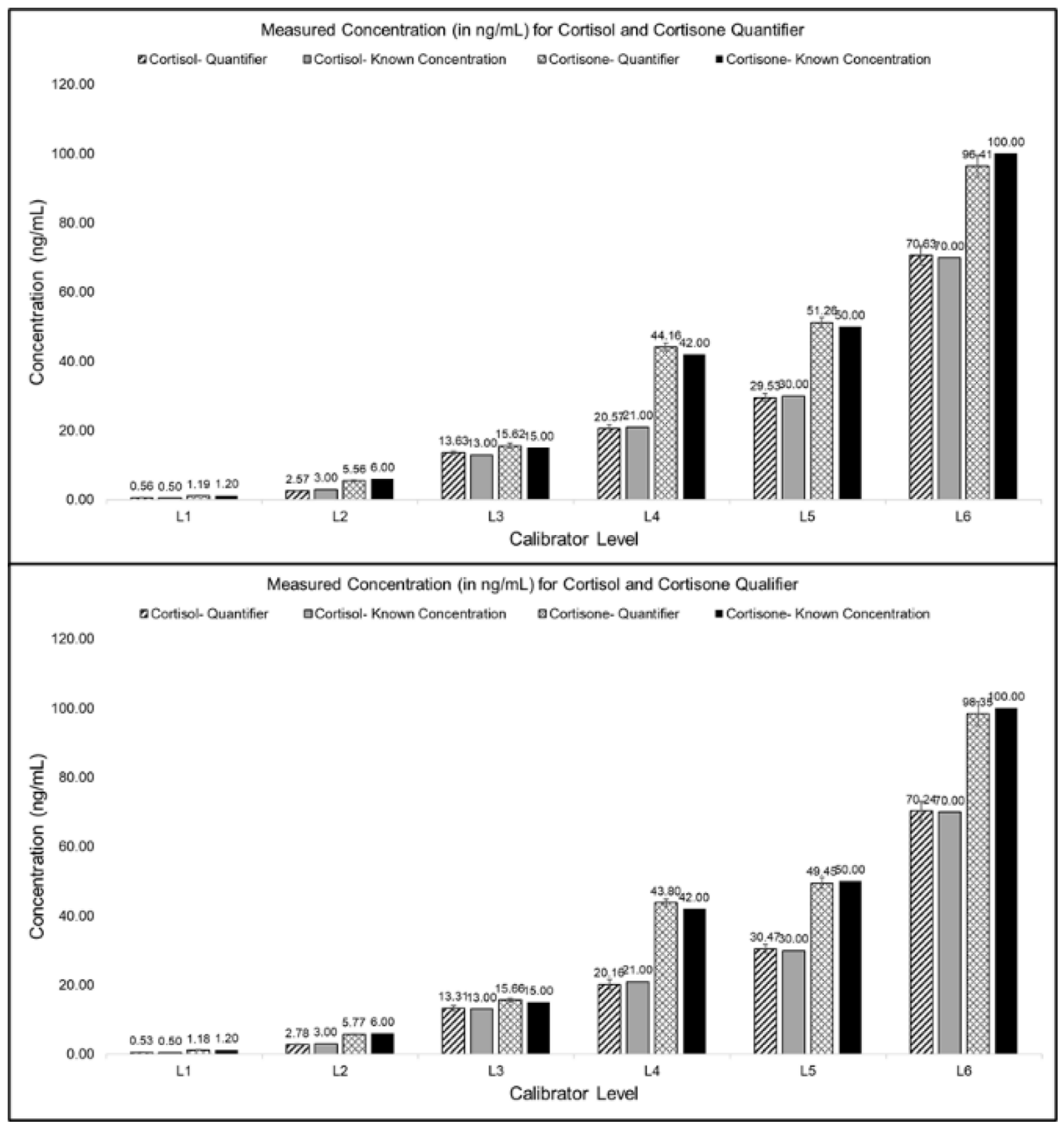

Fig. 3. Predicted and experimental concentrations for cortisol and cortisone in the 14-day accelerated stability study. While the predicted trend shows a much sharper decline, experimentally we found both analytes to be stable for a longer time than predicted.

room temperature) were measured and plotted for each run. The data obtained was first observed in JMP Pro 15.2 for outliers. For all six freeze-thaw cycles of both freezing temperatures, the concentration per calibrator level was first compared using a two-tailed Student's paired ttest $(\alpha=0.05)$ for the technical replicates. No significant difference was observed in the measured concentrations for either temperature across all calibrator levels. In addition, we used the connecting letter report generated by JMP to confirm that the measured calibrators were indeed significantly different from each other and the blank matrix. This is especially important in the context of the lowest-concentration calibrators (L1 and L2) that are only slightly above the background noise level. The connecting letter report indicated the significant difference among all calibrator levels and blanks and showed no significant difference for the same level across runs. This concludes that there is no degradation due to cryopreservation and thawing on the analytes and the same batch of manufactured calibrators can be used for multiple assays over time. The average concentration per calibrator level, per freeze-thaw cycle run is available on Supplemental Fig. S3.

\subsection{Sample carry-over}

To assess sample carryover, we first established a baseline signal for L1 (lowest calibrator level), followed by one L6 (highest calibrator) injection, followed by 3 immediate injections of L1. MRM analyses of both analytes showcased no statistically significant deviation in the observed L1 measurements from the L1 baseline, indicating that there was no carryover effect in any of the measurements carried out (data spread shown in Supplemental Fig. S4). Minimal carryover effect is desirable particularly if this method is to be implemented at a clinical setup. Performing blank washes after each patient sample injection would mean that fewer samples can be tested per plate to accommodate the wash wells. Our method integrates autosampler Needle Wash Solvent Chem- 


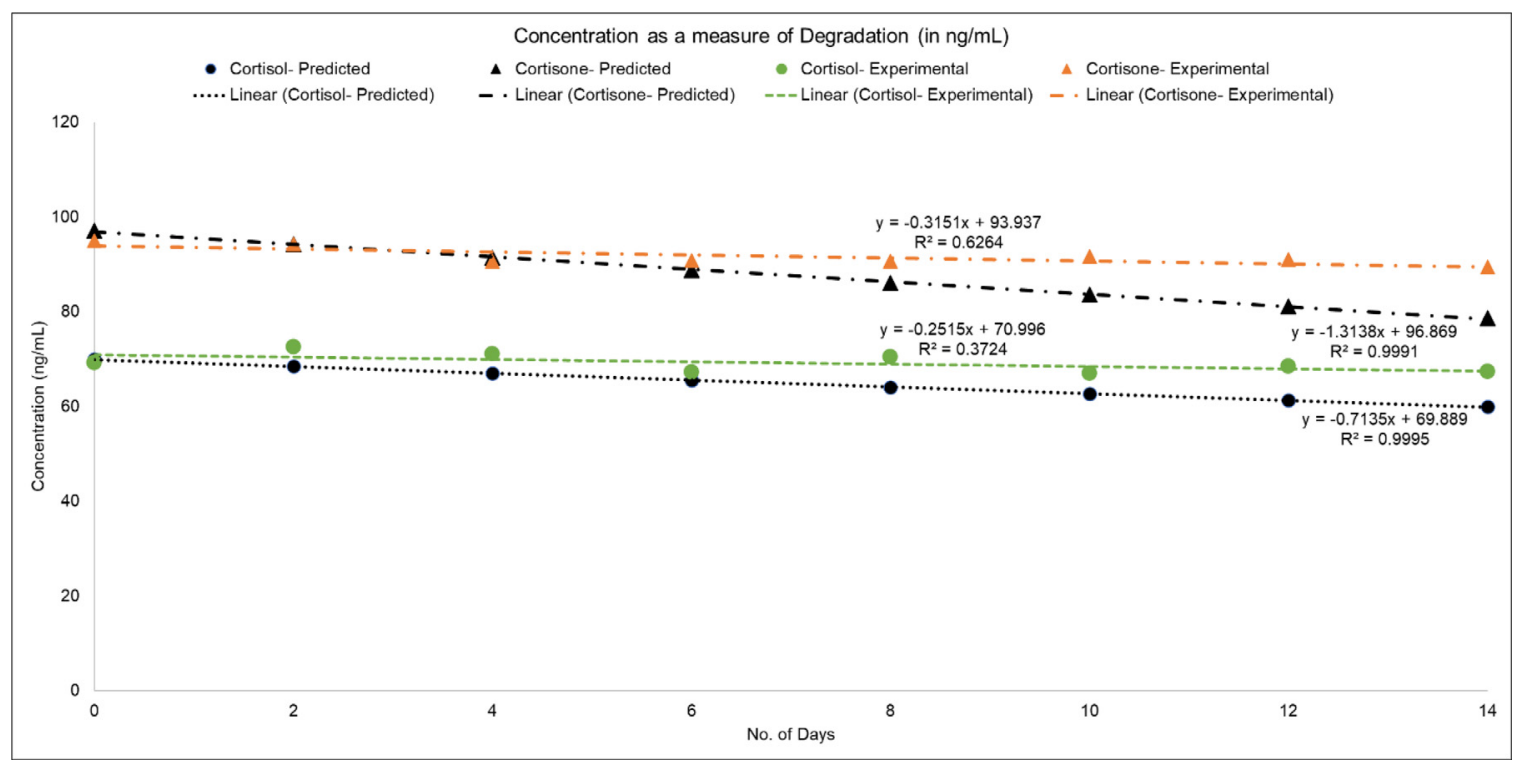

Fig. 4. JANUS ${ }^{\circledR}$ G3 Workstation (left) versus human (right) standard curves for cortisol and cortisone (qualifier and quantifier); $n=3$ per level per plate. The standard curve obtained from JANUS plating showed less spread in the data points compared to the human plate, although the assay linearity was measured at 0.99 and above for both.

istry (as described in the methods section), thus mitigating the sample carryover. This method can be easily transferred for many patient samples tests without the need for any blank insertion after each sample injection.

\subsection{Kit stability}

For our 14-day accelerated stability study (488 days extrapolated), we ran two plates per temperature point and duplicates per point. The data obtained was first converted to $\%$ change (in relation to Day $=0$ samples) and then normalized. For both $-20^{\circ} \mathrm{C}$ and $+30^{\circ} \mathrm{C}$ stress temperatures, it was difficult to obtain a conclusive trend for either analyte over the 14-day period. There was a significant difference between the concentration obtained for $\mathrm{D}=0$ and $\mathrm{D}=14$; however, there was no conclusive result for when this decline/degradation began to happen. To take a closer look at this, we ran the $D=0, D=2, D=4, D=6$ samples for all calibrators for $-20^{\circ} \mathrm{C}$ stress temperature in triplicate. For this run, we looked at the raw concentration values across the 6-days, as well as the $\%$ change and the normalized data. This data was then extrapolated to obtain a degradation equation that could be used to plot a trendline. The data obtained from our initial study was plotted against the predicted trendline from the extrapolated data. It was interesting to observe that while the extrapolated trend predicted a steady $2 \%$ decrease in cortisol concentration and a $4 \%$ decrease in cortisone concentration, for each day of the accelerated study, the true measured data (in duplicates, across 14-days) exhibited a much slower decline in concentration. Because the predicted values are extrapolated and the experimental values were in duplicates, Fig. 4 shows no error bars. We then performed a Tukey-Kramer HSD analysis to obtain any statistically significant difference for each level across the six days (measured in triplicates), hoping to reach a conclusion for the stability of the designed 'kit'. The generated connected letter report showed no significant difference in the means of the concentration measured (for both analytes) across the 4-days, stating that the analytes are stable for 8.5 months ( 256 days) at $-80^{\circ} \mathrm{C}$ storage. It is important to note that the reported decline is well-within our $20 \%$ tolerance for precision and accuracy, and since it is an accelerated study, the stability likely extends beyond the time points tested.

\subsection{Automation efficacy, precision, and accuracy}

Owing to the ease of sample preparation, we were able to quickly adapt the sample preparation protocol to the JANUS ${ }^{\circ}$ G3 workstation. The established automation protocol demonstrated the capability of completing a precision study on 48 samples in 4.93 minutes, compared to the 6-minute of human plating (human intervention and ondeck actions are illustrated in Supplemental Fig. S5). The reported RSquare value for the linear regression for an average of 3 automated runs was $\geq 0.99$, demonstrating that it possesses the required assay linearity. Moreover, automated sample runs reported a considerable improvement in precision (\%CV), showcasing a decreased spread in data points across the standard curve (particularly for the higher-concentration calibrators). Since we have already established the overall linearity and accuracy of this assay, we adhered to the use of data points spread in standard curves as a measure of improved precision (Fig. 5). The automated procedure made the entire assay just above 3-minutes for plating, compared to the human 6-minutes plating. It is important to note that human plating required re-capping the calibrator, QC, and sample vials, to prevent contamination, whereas all the vials could be kept open during the entire duration of plating on the JANUS deck. This action will prove particularly advantageous when translating this method at a high throughput laboratory or clinical setting. Because of the already established assay precision and accuracy, the automation step can be designed for single calibrator injections, leaving the user 81-wells open for at least 81-unknown samples testing in singlicate. Given our study utilizing a small volume $(20 \mu \mathrm{L})$ sample, small human error in pipetting can magnify, propagate and have a significant impact on the assay precision. That is one avenue where automation greatly helps due to its ability to accurately dispense small volume samples, minimizing error propagation.

\section{Conclusions}

The presented work successfully establishes an optimized LC-MS/MS method for the simultaneous detection of free cortisol-cortisone in saliva. Moreover, a quantification kit for the diagnostic evaluation of cortisol-cortisone was developed. Based on a wide number of assessments performed over the course of this work, the proposed LC-MS/MS 
Standard Curve: "Concentration vs Area Ratio" Source "ESI1" Component "Cortisol (363.1/120.9)" Internal Standard "Cortisol IS $y=0.13380 x+0.03198 \quad R^{2}=0.9945$ (ByArea, Linear, $1 / X$ \}

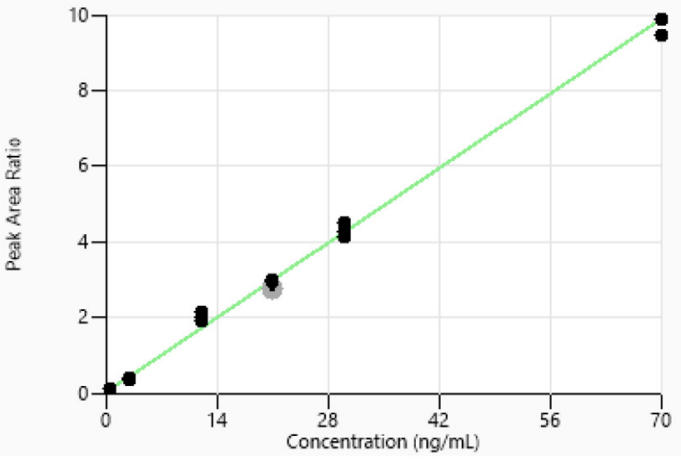

Standard Curve: "Concentration vs Area Ratio"

Source "ESI1" Component "Cortisone (361.1/121)" Internal Standard "Cortisone IS $y=0.04001 x+0,02826 \quad R^{2}=0.9964$ [ByArea, Linear, $1 / x$ ]

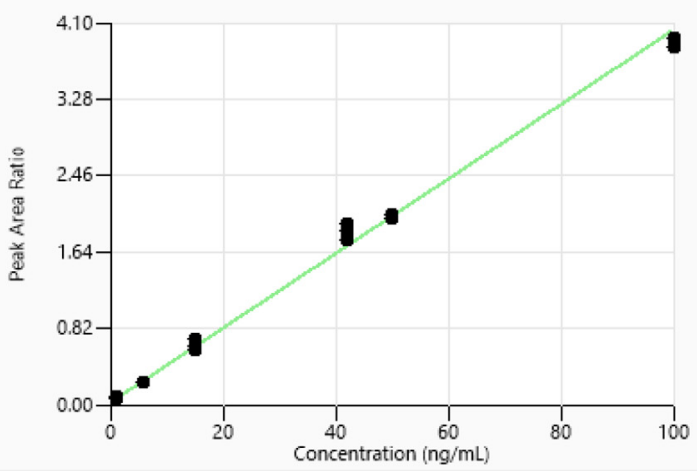

Standard Curve: "Concentration vs Area Ratio"

Source "ESI1" Component "Cortisol (363.1/120.9)" Internal Standard "Cortisol IS $y=0.14294 x+0.01131 \quad R^{2}=0.9904$ (ByArea, Linear, $1 / X$ )

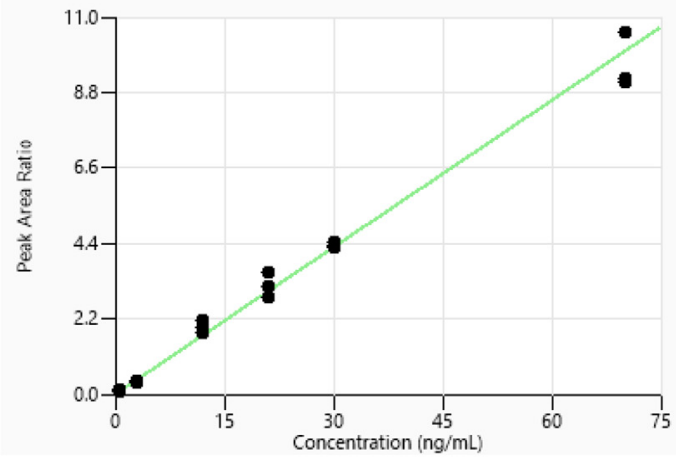

Standard Curve: "Concentration vs Area Ratio"

Source "ESI1" Component "Cortisone (361.1/121)" Internal Standard "Cortisone IS $y=0.04247 x+0.02918 \quad R^{2}=0.9971$ (ByArea, Linear, $1 / X$ )

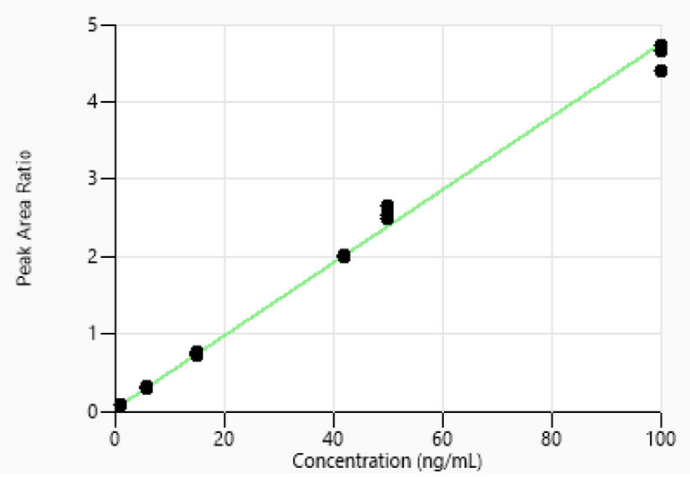

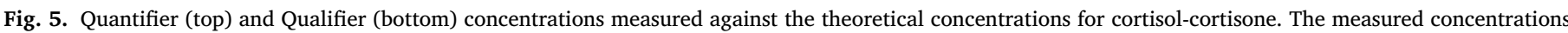
were used in the generation of the standard curves for all further tests.

method is rapid, sensitive, and precise. The methodology showcased the stability, linearity, and simplicity required for theoretical clinical use. When compared with previous reports, this method and quantification kit additionally showcased a heightened simplicity in the sample preparation process, owing to the lack of expensive and complex phase separation processes required in previous literature. Overall, this allowed for the full automation of the sample preparation process, furthering the usability of this work in the clinical setting. Pending further validation, this quantification kit may be used to provide valuable information in the clinical diagnostic realm for therapeutic monitoring of cortisol cortisone to elucidate potential adrenal dysfunctions in patients suffering from $\mathrm{CAH}$, Cushing's Syndrome, as well as other endocrine disorders.

\section{Declaration of interests}

The authors declare the following financial interests/personal relationships which may be considered as potential competing interests:

Anubhav Tripathi reports financial support was provided by PerkinElmer Inc. Anubhav Tripathi reports a relationship with PerkinElmer Inc that includes: consulting or advisory.

\section{Acknowledgments}

We would like to thank Kathryn Sherlock for helping with the automation troubleshooting, and Rudra Maharaj for his valuable insight during the initial phase of this study. We would also like to thank Celeste Welch and Lindsay Schneider for their valuable scientific feedback throughout the study. A.T. is a paid scientific advisor/consultant and lecturer for PerkinElmer.

\section{Supplementary materials}

Supplementary material associated with this article can be found, in the online version, at doi:10.1016/j.slast.2022.01.006.

\section{References}

[1] Timmermans S, Souffriau J, Libert C. A general introduction to glucocorticoid biology. Front Immunol 2019;10:1545.

[2] Stephens MAC, Wand G. Stress and the HPA axi: Role of glucocorticoids in alcohol dependence. Alcohol Res 2012.

[3] Ehlert U, Gaab J, Heinrichs M. Psychoneuroendocrinological contributions to the etiology of depression, posttraumatic stress disorder and stress-related bodily disorders: the role of the hypothalamus-pituitary-adrenal axis. Biol Psychol 2001;57:141-52.

[4] Nicolaides NC, Galata Z, Kino T, Chrousos GP, Charmandari E. The human glucocorticoid receptor: molecular basis of biologic function. Steroids 2010;75:1-12.

[5] Ramamoorthy S, Cidlowski JA. Corticosteroids: mechanisms of action in health and disease. Rheum Dis Clin 2016;42:15-31.

[6] Kuo T, McQueen A, Chen T-C, Wang J-C. Regulation of glucose homeostasis by glucocorticoids. Glucocorticoid Signal 2015:99-126.

[7] Exton J. Regulation of gluconeogenesis by glucocorticoids. Monogr Endocrinol 1979:12:535-46.

[8] Olgemöller B, Roscher AA, Liebl B, Fingerhut R. Screening for congenital adrenal hyperplasia: adjustment of 17-hydroxyprogesterone cut-off values to both age and birth weight markedly improves the predictive value. J Clin Endocrinol Metabol 2003;88:5790-4. doi:10.1210/jc.2002-021732.

[9] New MI. Diagnosis and management of congenital adrenal hyperplasia. Annu Rev Med 1998;49:311-28.

[10] Yamamoto T. Latent adrenal insufficiency: concept, clues to detection and diagnosis. Endocr Pract 2018;24:746-55.

[11] Mitchell IC, et al. Subclinical Cushing's syndrome" is not subclinical: improvement after adrenalectomy in 9 patients. Surgery 2007;142 900-905. e901.

[12] Do Yup Lee EK, Choi MH. Technical and clinical aspects of cortisol as a biochemical marker of chronic stress. BMB reports 2015;48:209.

[13] Seger C, Salzmann L. After another decade: LC-MS/MS became routine in clinical diagnostics. Clin Biochem 2020;82:2-11. 
[14] Jung C, Greco S, Nguyen HH, Ho JT, Lewis JG, Torpy DJ, Inder WJ. Plasma, salivary and urinary cortisol levels following physiological and stress doses of hydrocortisone in normal volunteers. BMC Endocrine Disord 2014;14(1):1-10.

[15] Tudela E, Muñoz G, Muñoz-Guerra JA. Matrix effect marker for multianalyte analysis by LC-MS/MS in biological samples. J Chromatogr B 2012;901:98-106.

[16] Papanicolaou Dimitris A, et al. Nighttime salivary cortisol: a useful test for the diagnosis of Cushing's syndrome. J Clin Endocrinol Metabol 2002;87(10):4515-21.

[17] Bacila Irina, et al. Measurement of salivary adrenal-specific androgens as biomarkers of therapy control in 21-hydroxylase deficiency. J Clin Endocrinol Metabol 2019;104.12:6417-29.

[18] Pasquali Renato, et al. Cortisol and ACTH response to oral dexamethasone in obesity and effects of sex, body fat distribution and dexamethasone concentrations: a dose-response study. J Clin Endocrinol Metabol 2002;87.1:166-75.

[19] Ospina Naykky Singh, et al. ACTH stimulation tests for the diagnosis of adrenal insufficiency: systematic review and meta-analysis. J Clin Endocrinol Metabol 2016;101.2:427-34.

[20] Hannibal KE, Bishop MD. Chronic stress, cortisol dysfunction and pain: a psychoneuroendocrine rationale for stress management in pain rehabilitation. Phys Ther 2014;94(12):1816-25.

[21] Bakusic J, De Nys S, Creta M, Godderis L, Duca RC. Study of temporal variability of salivary cortisol and cortisone by LC-MS/MS using a new atmospheric pressure ionization source. Sci Rep 2019;9(1):1-12.

[22] Magda B, Dobi Z, Mészáros K, Szabó É, Márta Z, Imre T, et al. Charged derivatization and on-line solid phase extraction to measure extremely low cortisol and cortisone levels in human saliva with liquid chromatography-tandem mass spectrometry. J Pharm Biomed Anal 2017;140:223-31.

[23] Antonelli G, Ceccato F, Artusi C, Marinova M, Plebani M. Salivary cortisol and cortisone by LC-MS/MS: validation, reference intervals and diagnostic accuracy in Cushing's syndrome. Clin Chim Acta 2015;451:247-51.

[24] Fustinoni S, Polledri E, Mercadante R. High-throughput determination of cortisol, cortisone and melatonin in oral fluid by on-line turbulent flow liquid chromatography interfaced with liquid chromatography/tandem mass spectrometry. Rapid Commun Mass Spectrom 2013;27(13):1450-60.

[25] Niewoehner, C. B. (2004). 5. In Endocrine pathophysiology (Second, pp. 85-105). essay, Hayes Barton Press.

[26] Stewart PM, Corrie JET, Shackleton CHL, Edwards CRW. Syndrome of apparent mineralocorticoid excess. A defect in the cortisol-cortisone shuttle. J Clin Invest 1988;82(1):340-9.

[27] Casals G, Hanzu FA. Cortisol measurements in Cushing's syndrome: immunoassay or mass spectrometry? Ann Labor Med 2020;40(4):285-96.

[28] Chabre O. The difficulties of pseudo-Cushing's syndrome (or "non-neoplastic hypercortisolism"). Ann Endocrinol 2018;79(3):138-45.

[29] Raff H, Phillips JM. Bedtime salivary cortisol and cortisone by LC-MS/MS in healthy adult subjects: evaluation of sampling time. J Endocr Soc 2019;3(8):1631-40.

[30] Erickson D, Singh RJ, Sathananthan A, Vella A, Bryant SC. Late-night salivary cortisol for diagnosis of Cushing's syndrome by liquid chromatography/tandem mass spectrometry assay. Clin Endocrinol 2012;76(4):467-72. 